Revista de la Facultad de Ingeniería Industrial

16(1): 9-16 (2013) UNMSM

ISSN: 1560-9146 (Impreso) / ISSN: 1810-9993 (Electrónico)

\title{
Sistema ISO 9001:2008. Experiencia de implementación en una pyme de confección de ropa industrial en el Perú, con énfasis en producción
}

\begin{abstract}
RESUMEN
Aplicar un Sistema de Gestión de Calidad (SGC) en una pequeña y microempresa (pyme) es una experiencia valiosa y un ejemplo para otras pymes en el Perú, es por ello, que se describe esta experiencia que obedece a un trabajo realizado para implementar el SGC ISO 9001:2008, con énfasis en producción, en una pequeña empresa de confección de ropa industrial, siendo esta, la primera del país en certificar en dicho rubro. El artículo señala los requisitos necesarios y el proceder adecuado que deben seguir las pymes de confección de ropa industrial para lograr una implementación exitosa en sus organizaciones y obtener la certificación.
\end{abstract}

Palabras clave: SGC, calidad, certificación auditoria, procesos

ISO 9001:2008 SySTEM. IMPLEMENTATION EXPERIENCE IN A SMALL AND MEDIUM ENTERPRISE OF INDUSTRIAL CLOTHING IN THE Peru, WITH EMFHASIS ON PRODUCTION

\section{ABSTRACT}

To apply a Quality Management System (QMS) in small business, is a worth experience and also it is an example for others in Peru. This is the reason why we describe this experience that is due to a work done to implement the QMS ISO 9001:2008 with an emphasis on production, in a small company of industrial garment, being this, the first in the country to certify in that field. The article points out the requirements and the proper procedure to be followed by a small industrial clothing company to achieve a successful implementation in their organizations and obtain the certification.

Keywords: QMS, Quality, certificate, audit, process

\section{INTRODUCCIÓN}

Si bien es cierto que existen empresas extranjeras dedicadas a la confección de ropa industrial y que cuentan con la certificación ISO 9001, según la lista publicada por el Centro de Desarrollo Industrial (CDI) [1], son solo tres las empresas de confecciones de ropa en el Perú que cuentan con dicha certificación. De estas tres, dos son grandes empresas y la tercera es una pyme cuya experiencia es la que se hace referencia en este artículo, siendo la única empresa dedicada a la confección de ropa industrial en el Perú que cuenta con esta certificación.

Actualmente la mayoría de empresas de confecciones de ropa industrial en el país no operan con mecanismos de aseguramiento de la calidad y mejora continua en sus procesos, como es el ISO 9001, esto les resta competitividad frente a los productos provenientes de empresas del extranjero, los cuales aplican SGC eficientes y eficaces, reconocidos internacionalmente. Estas empresas nacionales carecen de los conocimientos acerca de los lineamientos de la norma ISO 9001 y de cómo hacer para aplicarlos a sus organizaciones.

Es muy importante que las pymes en el Perú puedan lograr estándares de calidad competitivos, con gran fortalecimiento organizacional y para así poder consolidarse en el mercado nacional e incursionar satisfactoriamente en el internacional.

\section{BASES TEÓRICAS}

\subsection{Enfoque por procesos}

El proceso representa un sistema particular, que opera dentro de un sistema general, cuyas actividades interactúan para obtener un resultado de mayor valor que al principio. Es importante su identificación, midiéndolos y controlándolos para asegurar un buen desempeño (gestión por procesos). La satisfacción del cliente dependerá mucho de cómo se desarrollan cada uno de los procesos.

Al iniciar la implementación de un SGC ISO 9001, las organizaciones deben reestructurarse hacia un enfoque por procesos y estos deben ser administrados adecuadamente, midiendo su desempeño por medio de indicadores claves, con el fin de tomar las

* Ingeniero Industrial. Jefe de Producción de TREXSA. E-mail: raul jesus1@hotmail.com ** Ingeniero Industrial. Docente Asociado de la UNMSM. E-mail: mparragav@hotmail.com 
acciones correctivas apropiadas y la consecuente mejora de los mismos y de toda la empresa.

Procesos que crean valor [2], están relacionados directamente con la creación de productos y servicios y tiene mucho que ver con la satisfacción del cliente, repercutiendo en los objetivos de la empresa. Están conformados generalmente por diseño, producción/entrega y demás procesos críticos.

Procesos de apoyo, por lo general no agregan valor de manera directa al producto o servicio, sin embargo, son de gran importancia para los procesos de creación de valor.

Propietarios de proceso, según Summers [3] "es el responsable de asegurarse de que un proceso se realice de tal manera que produzca satisfacción en el cliente. Estos individuos pueden, y tienen las facultades necesarias para hacer cambios al proceso".

\section{METODOLOGÍA}

Las actividades para lograr el objetivo de implementar el SGC en la organización, son:

1. Comprometer a la alta dirección, quien debe proporcionar los recursos necesarios, predicar con el ejemplo y ser líder del sistema. A su vez definir la política de calidad de la empresa, estableciendo los objetivos de calidad.

2. Obtener información relacionada al SGC ISO 9001 (biblioteca, Internet, personas de experiencia en el tema).

3. Recabar información en Internet, relacionado a las otras empresas en el extranjero que han certificado con ISO 9001, en el rubro de la confección de ropa industrial.

4. Coordinar con el área de recursos humanos para capacitar al personal, en relación a los requerimientos de la norma ISO 9001 y la adquisición de nuevas competencias.

5. Programar reuniones con cada líder de proceso, brindando información en relación al proceso de implementación y su importancia.

6. Identificar los procesos del sistema y asignar responsabilidades a cada líder de proceso, con la finalidad de obtener propuestas de documentación, formato de registros, los cuales son analizados para su posterior implementación.

7. Coordinar con cada líder de proceso para reorganizar las actividades, con la ayuda de diagramas de flujo.
8. Revisar los avances logrados durante el proceso de implementación, realizando ajustes.

9. Programar y efectuar auditorías internas en coordinación con los auditores internos, capacitados para tal fin. Analizar los resultados de la auditoría.

10.Coordinar con la alta dirección y la empresa certificadora, para determinar la fecha de realización de la auditoría de certificación.

11. Obtener conclusiones, luego de obtener la certificación.

\subsection{Obtención de datos}

Las listas de verificación, como se observa en la Tabla 1, son las herramientas principales para la obtención de los datos, las cuales mostrarán el estado inicial de la empresa en relación a los requerimientos de la norma ISO 9001. En este punto, es cuando se entrevista a los líderes de procesos y parte de sus empleados.

Dicho instrumento está conformado por una serie de preguntas abiertas estructuradas no generales, los cuales están relacionados con los requerimientos que exige la norma.

\subsection{Administración de actividades}

Una administración adecuada de las actividades, se logra con la ayuda del estudio de métodos, el cual registra las labores realizadas, ejecutando un análisis sistemático, facilitando la generación de ideas y buscando nuevas formas de efectuar el trabajo, incrementando la productividad.

\subsection{El factor humano y su papel determinante en el logro de objetivos}

La participación del empleado es de suma importancia para la empresa, por ello, todas las áreas deben recibir capacitación constante en temas relacionados a la calidad. El trabajador conoce con mayor detalle la labor asignada, pudiendo intervenir eficientemente en la mejora de la misma, sugiriendo cambios en los procesos para mejorar la calidad [4]. Sin embargo, para que aquello resulte un éxito, se debe proveer los recursos necesarios para un buen desempeño y a su vez disponer de experiencias en relación a las actividades ejecutadas en los diferentes procesos.

\subsection{Documentación del SGC ISO $9001: 2008$}

La norma es práctica al permitir a las organizaciones incluir en sus sistemas la documentación mínima necesaria y poder demostrar una eficaz planificación, ejecución, control de procesos y mejora. (Ver Figura 1). 
Tabla 1. Lista de verificación

\begin{tabular}{|l|l|l|l|l|}
\hline \multicolumn{5}{|c|}{ LISTA DE VERIFICACIÓN } \\
\hline Área: \\
Nombre: & \multicolumn{2}{|c|}{ Fecha: } \\
\hline 4 Sistema de Gestión de la Calidad
\end{tabular}

${ }^{*} \mathrm{P}=$ parcial, se debe especificar en observación el avance alcanzado.

Nota 1: El término procedimiento documentado, significa que el procedimiento se ha establecido, documentado, implementado y mantenido. Un solo documento puede incluir los requisitos para uno o más procedimientos. Un requisito relativo a un procedimiento documentado puede cubrirse con más de un documento.

Nota 2: La extensión de la documentación del SGC puede diferir de una organización a otra debido a:

- El tamaño de la organización y el tipo de actividades,

- La complejidad de los procesos y sus interacciones, y

- La complejidad del personal.

Nota 3: La documentación puede estar en cualquier formato o tipo de medio.

Fuente: Elaboración propia.

Figura 1. Documentación del SGC ISO 9001:2008.

Fuente: ISO 9001:2008.

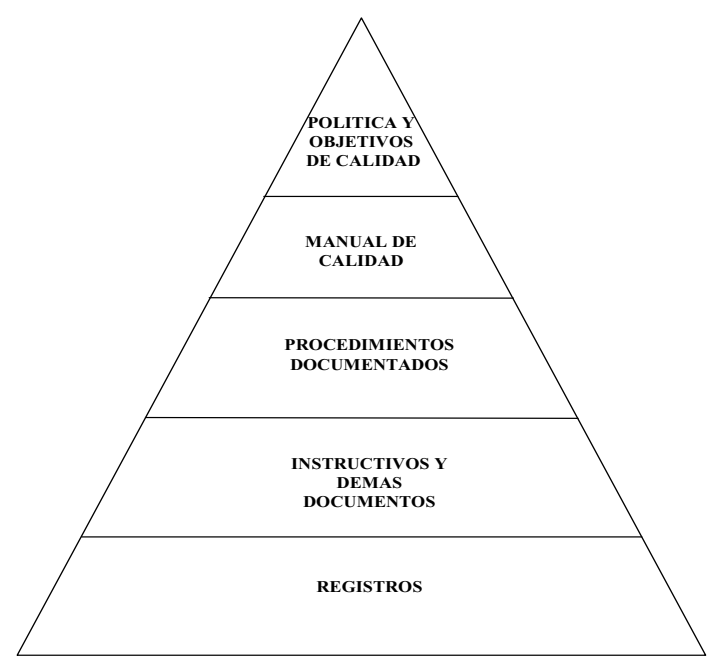




\subsection{Determinación del equipo responsable}

La alta dirección, al haber tomado la decisión de dar inicio al proceso de implementación, nombra al Representante de la Dirección (RED), quien ostenta la máxima responsabilidad y selecciona un equipo de trabajo. El equipo se compone por personal de la misma organización, mostrado en la Tabla 2.

La asignación de tareas es distribuida a cada miembro del equipo de trabajo, por el RED. Entre las principales tareas encomendadas a cada uno de los miembros, se tiene lo siguiente:

- Recopilar información en relación a todas las actividades desarrolladas en el área a cargo.

- Dialogar y concientizar al personal del área a cargo, acerca de la importancia de su participación y cooperación durante todo el proceso de implementación del SGC.

- Informar al personal lo relacionado a la norma ISO 9001.

- Efectuar un informe escrito al RED sobre lo desarrollado en el área asignada.

\subsection{Diagnóstico}

El RED o auditor líder, es quien efectúa el diagnóstico en relación a la norma ISO 9001, para ello utiliza la Lista de Verificación (Figura 1), donde se toma en cuenta todos los puntos de la norma. También puede ser un auditor externo el que efectúe el diagnóstico, en el caso que no se cuente con el personal de suficiente experiencia en cuanto a interpretación de la norma.

A cada pregunta de la Lista de Verificación se muestra tres respuestas y una columna de observaciones. Estas son:

Sí Representa el cumplimiento del punto de la norma, sometida a interrogación.

No Significa que no cumple con el punto de la norma, sometida a interrogación.

P Representa un cumplimiento parcial del punto de la norma, sometida a interrogación.

Tabla 2. Perfil del RED y del equipo de trabajo.

\begin{tabular}{|c|c|c|}
\hline \multicolumn{3}{|c|}{ RED } \\
\hline \multicolumn{3}{|c|}{ 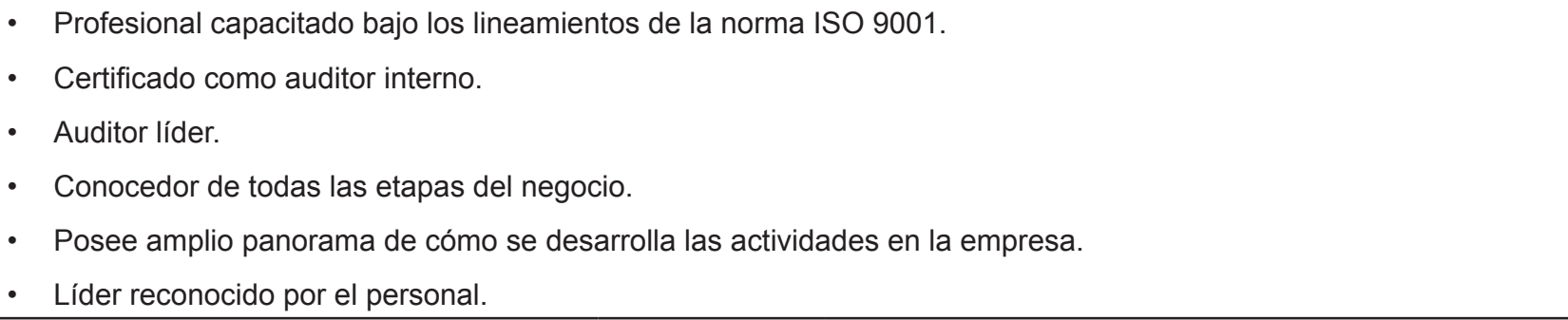 } \\
\hline \multicolumn{3}{|c|}{ Equipo de trabajo } \\
\hline \multirow{4}{*}{$\begin{array}{l}\text { - Representan los puntos trascendentales } \\
\text { de la organización, en relación a las } \\
\text { actividades desarrolladas. } \\
\text { - Poseen cualidades para el trabajo en } \\
\text { equipo. } \\
\text { - Capacitados en ISO } 9001 \\
\text { - Certificados como auditor interno. }\end{array}$} & Miembro 1: & $\begin{array}{l}\text { - Conoce las actividades desarrolladas en el área de } \\
\text { producción y su interacción con las demás. } \\
\text { - Representado por el jefe de producción. }\end{array}$ \\
\hline & Miembro 2: & $\begin{array}{l}\text { - Conoce las actividades desarrolladas en el área de } \\
\text { ventas y su interacción con las demás. } \\
\text { - Representado por el jefe de ventas. }\end{array}$ \\
\hline & Miembro 3: & $\begin{array}{l}\text { - Conoce las actividades desarrolladas en el área de } \\
\text { logística y su interacción con las demás. } \\
\text { - Representado por el jefe de logística. }\end{array}$ \\
\hline & Miembro 4: & $\begin{array}{l}\text { - Conoce las actividades desarrolladas en el área } \\
\text { administrativa y su interacción con las demás. } \\
\text { - Representado por el subgerente. }\end{array}$ \\
\hline
\end{tabular}

Fuente: Elaboración propia. 


\subsection{Implementación}

Consiste en desempeñar las actividades, en cada proceso identificado, según la documentación elaborada para el SGC, los cuales se aplican desde el momento mismo de su creación. Cuando se realizan ajustes, ya sea de formatos para registros, instructivos o procedimientos, estos son regulados con la participación del trabajador hasta lograr un eficiente uso de los mismos. Durante la implementación se da comienzo a la aplicación de los registros según los formatos elaborados y de acuerdo a lo señalado en los procedimientos. Una vez transcurrido un periodo de tiempo, se procede a establecer los objetivos, indicadores y metas, de acuerdo a la información obtenida de los registros.
EI RED y cada miembro del equipo de trabajo, deben monitorear el avance de la implementación y orientar los esfuerzos por mantener lo implementado.

\subsection{Identificación de procesos}

Para determinar los procesos necesarios para el SGC y su aplicación en la organización, es muy importante conocer todas las actividades presentes desde el primer contacto con el cliente, hasta la atención del servicio, al final de las operaciones. Todas las actividades desarrolladas en cada proceso, siguen los lineamientos del PHVA (Planear, Hacer, Verificar y Actuar). Como ejemplo se detalla el proceso de control de calidad. Figura 2.

Figura 2. Proceso de control de calidad en la Empresa TREXSA E.I.R.L.

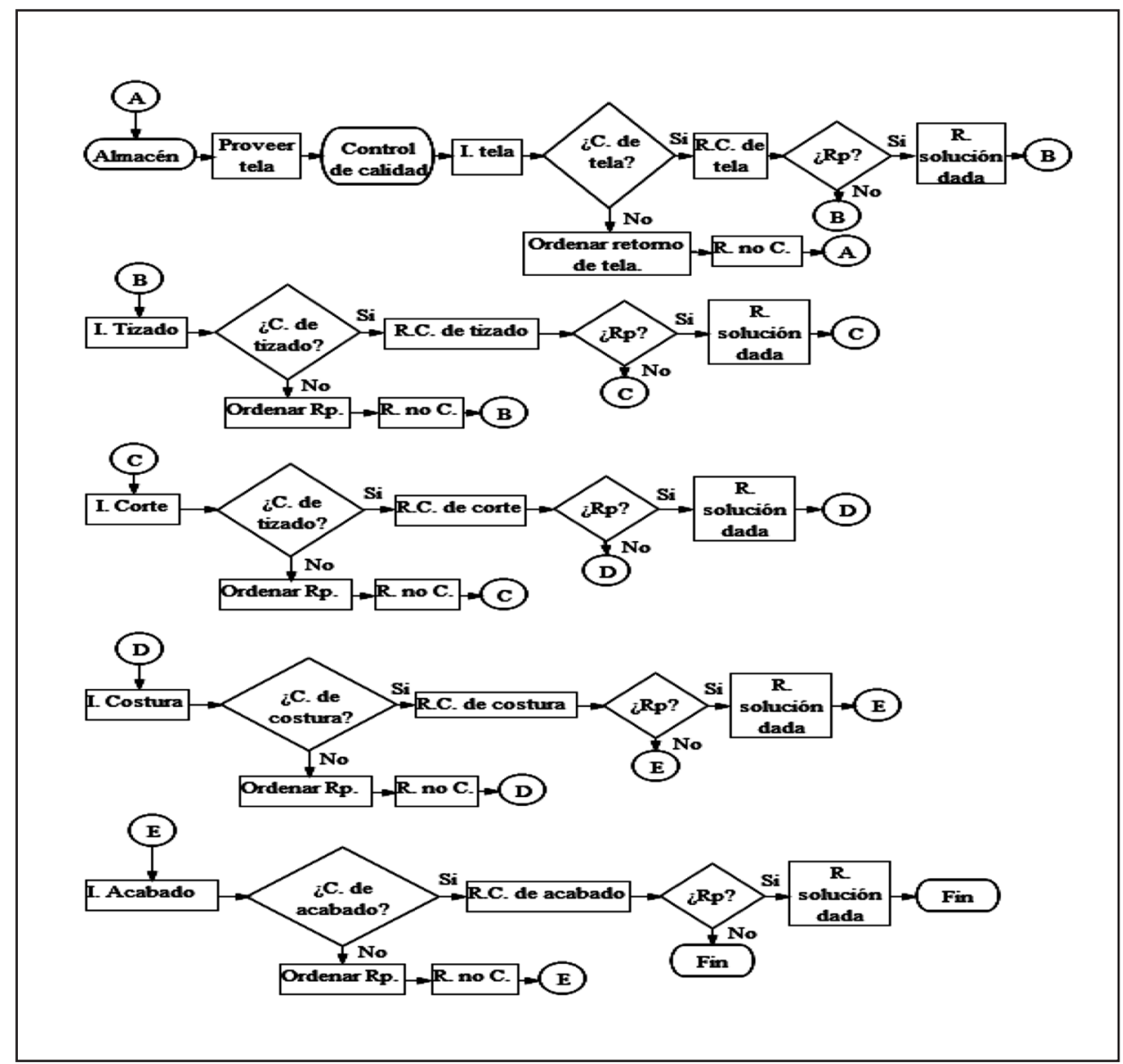

Donde:
C Conformidad
I Inspeccionar
R Registrar

Rp. Reproceso

Fuente: Elaboración propia. 


\subsection{Esquema general del proceso de implementación}

En la Figura 3 se muestra en grandes lineamientos el esquema a seguir para la implementación del SGC ISO 9001:2008.

Figura 3. Esquema general del proceso de implementación del sistema de gestión de calidad ISO 9001:2008.

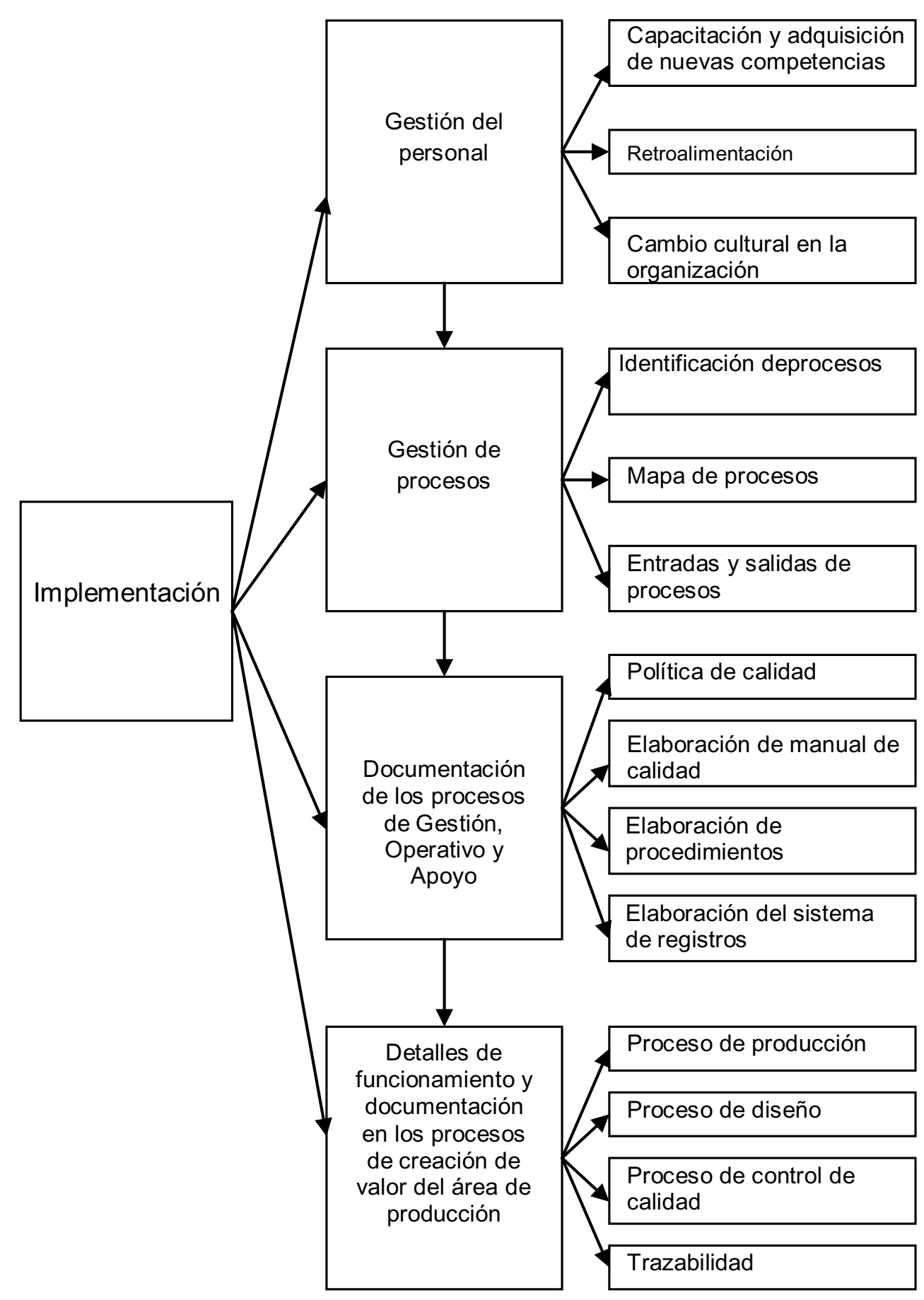

Fuente: Elaboración propia. 


\subsection{Auditorías internas}

Es un mecanismo de control para la alta dirección y poder medir la eficacia del sistema, constituyen una herramienta de prevención de defectos y una ayuda para detectar oportunidades de mejora dentro de la organización. Por tanto, de acuerdo a la certificación ISO 9001:2008, las auditorías internas representan un paso previo y necesario para saber si la empresa se encuentra lista para solicitar una auditoría de certificación.

Estas auditorías son realizadas por la misma empresa con la participación del personal previamente capacitado como auditores internos, o por una empresa externa, especializada en el tema. Se distribuye adecuadamente a los auditores internos, por medio del plan de auditorías, de tal manera que cada uno de ellos no audita a los procesos donde habitualmente se desempeñan, garantizando la imparcialidad en los resultados.

\subsection{Auditoría de certificación}

EI RED coordina la programación de la auditoría con la empresa certificadora, quien envía el plan de auditoría. Al inicio de la auditoría el RED presenta a cada uno de los líderes de los procesos que son auditados. La empresa certificadora ejecuta revisiones a la documentación del sistema para verificar que se cumplen los requerimientos de la norma, por medio de entrevistas al personal que confirmen que las actividades son realizadas de manera controlada. Durante el desarrollo de las auditorías surgieron no conformidades del sistema de calidad, los cuales fueron menores, no presentándose ninguna conformidad mayor y resueltos en un lapso de tiempo no mayor a tres meses. Después de realizadas las auditorías de certificación y dar solución a las inconformidades, el organismo certificador otorgó el certificado ISO 9001:2008 siguiendo los procedimientos y disposiciones planteados en la presente investigación, en un plazo aproximado de dos meses.

\subsection{Mantenimiento y mejora continua del SGC}

Una vez implementado y certificado el SGC, el siguiente paso ahora es mantenerlo, verificando constantemente que se esté utilizando la documentación establecida de acuerdo a los procedimientos y el fin para el cual fueron creados. El RED es el responsable del mantenimiento del sistema y gestiona las actividades necesarias para el sostenimiento del mismo. Es trascendental, la realización de auditorías internas, comité de calidad, revisión por la dirección y la participación activa de la alta dirección, el compromiso constante de los líderes de procesos y la constante comunicación en todos los niveles de la compañía. Finalmente, la empresa certificadora efectuará una auditoría de seguimiento una vez por año, como verificación del mantenimiento del SGC.

\section{CONCLUSIONES}

Contar con un SGC distingue a la empresa por su capacidad de brindar consistentemente productos que satisfagan los requerimientos del cliente y que asegure su aceptación a partir de una constante adecuación de sus procesos.

Toda empresa que quiera asumir nuevos retos y acceder a un mayor crecimiento debe buscar un instrumento que le permita afrontarlos con eficiencia y el ISO 9001:2008 es una herramienta de gestión internacional sólida.

A continuación se señalan las conclusiones del estudio y sus recomendaciones.

a. El funcionamiento eficaz del sistema solo es posible al operar en una gestión por procesos, para ello se identificaron todos los necesarios, señalando a los clientes y proveedores internos, trabajando bajo los criterios del círculo de calidad PHVA, que hacen posible la mejora continua del sistema.

b. En cada proceso, se especificaron los objetivos de calidad, los indicadores a utilizar y la meta hacia donde deben llegar los resultados, producto del análisis de la información extraída de los registros, enfocado siempre en la mejora continua del SGC.

c. Se han identificado los procesos de creación de valor, los cuales son cruciales en el aseguramiento de la calidad del producto.

d. Se establecieron los procedimientos exigidos por la norma y se crearon otros, considerados importantes para la estandarización de las diferentes actividades desplegadas en la organización, sirviendo de guía para el trabajador en sus labores diarias.

e. Los registros constituyen una base esencial en las decisiones que se tomen para la mejora continua del sistema. Para tal fin, se crearon una serie de formatos utilizados en la obtención de información asignados en los puntos críticos del sistema.

f. La participación del personal, con convicción, representa uno de los grandes pilares para la implementación exitosa del SGC. Para este 
propósito, se efectúo una apropiada capacitación y retroalimentación, cimentando una cultura de calidad en la organización y fortaleciendo la comunicación entre todos los integrantes.

g. Es trascendental mantener el SGC siempre activo y dinámico, con un seguimiento concienzudo del RED y el compromiso de la alta dirección, donde cada uno de los integrantes de la organización es consciente de su importancia.

\section{RECOMENDACIONES}

a. Es aconsejable la utilización de ayudas visuales, colocados en los diferentes ambientes de la organización, conteniendo información clave de los puntos críticos de los procesos, con el fin de facilitar al personal en sus labores cotidianas y minimizar la aparición de no conformidades.

b. Es importante contar con reuniones constantes entre los líderes de procesos, jefes de área, RED y la alta dirección, en relación al seguimiento del logro de objetivos, analizando la información recabada de los registros.

c. Es conveniente tener un ambiente de trabajo amplio, que facilite las labores, donde no existan lugares hacinados. La implementación del SGC no solo debe significar tener una excelente documentación, sino que, también la organización debe mostrar un aspecto que transmita calidad y limpieza en todas sus instalaciones.

d. Es muy importante la constante comunicación entre la organización y el cliente externo, para saber sus inquietudes y conocer el grado de satisfacción que tienen en la actualidad, aplicando adecuadamente las acciones correctivas ante cualquier no conformidad presentada.

e. Es elemental poseer un buen sistema de cómputo e informático, que agilicen las labores cotidianas de la organización.

f. La seguridad y salud ocupacional del trabajador deben representar una parte primordial en el eficiente desempeño del SGC.

\section{REFERENCIAS BIBLIOGRÁFICAS}

[1] http://www.cdi.org.pe/asistencia empcertificadas.htm

[2] Evans, J. R., Lindsay, W. M. (2005). Administración y control de la calidad. México, International Thomson Editores S.A., pp. 316-317.

[3] Summers, Donna C. S. (2006). Administración de calidad. México, Pearson Educación, $202 \mathrm{pp}$

[4] Besterfield, D. H. (2009). Control de calidad. México, Pearson Educación, pp. 41-45

[5] International organization for standardization, 2008. (Introduction and support package: Guidance on the documentation requirements of ISO 9001-2008), http://www.iso.org/ iso/iso_catalogue/management_and leadership_standads/quality_management/ iso_9001_2008/guidance_on_the _ documentation_requirements _of iso_9001_2008.htm, visitado el 15/12/2011. 